\title{
Magnetic resonance diffusion-weighted imaging in diagnostics of primary fallopian tube carcinoma - is it useful?
}

\author{
Laretta Grabowska-Derlatka ${ }^{1 A, B, D, E, F}$, Wojciech Szeszkowski ${ }^{1 A, C}$, Jacek Sieńko ${ }^{2 B, F}$, Paweł Derlatka ${ }^{2 A, B, D, E}$ \\ ${ }^{1} 2^{\text {nd }}$ Department of Radiology, Medical University of Warsaw, Poland \\ ${ }^{2} 2^{\text {nd }}$ Department of Obstetrics and Gynaecology, Medical University of Warsaw, Poland
}

\section{Abstract}

Purpose: Primary fallopian tube carcinoma (PFTC) is the rarest form of female genital malignancy. The imaging applied for suspected adnexal masses includes transvaginal ultrasound (US), computed tomography (CT), and magnetic resonance imaging (MRI), but the vast majority of PFTC is recognised intraoperatively.

Material and methods: The study group consisted of seven women with postoperatively histopathological diagnosis of PFTC. To recognise characteristic findings for PFTC, retrospective analysis of preoperative MRI was performed. All patients underwent MRI of the pelvis and abdomen using a 1.5T MR system. Based on the results of the above imaging, suspected adnexal masses were recognised. MRI protocol contained T2-weighted images, fat-suppressed T2-weighted, T2-TIRM, DW EPI, pre- and postcontrast dynamic 3D T1 GRE in transverse orientation, with diffusion weightings of $0,50,100,150,200,400,800$, and $1200 \mathrm{~s} / \mathrm{mm}^{2}$. Regions of interest were outlined by a radiologist, who documented the character of adnexal masses on diffusion-weighted (DW) images and apparent diffusion coefficient (ADC) maps.

Results: In all seven patients with PFTC unilateral tumour was found. On all DW images (with $b$ values of 0, 50, 100, 150, $200,400,800$, and $1200 \mathrm{~s} / \mathrm{mm}^{2}$ ) the mean signal intensities of solid parts of tumour were significantly higher than the mean signal intensities of normal ovarian tissue $(p=0.0001)$. There were no statistically significant differences between eight $b$ values applied for ADC calculations.

Conclusions: Preoperative diagnostics of PFTC is difficult and mainly based on morphological features. Previous research did not show characteristics of PFTC in post-contrast dynamic imaging. In our material a clear increasing of signal intensity in DW imaging occurred independently of the $b$ value.

Key words: primary fallopian tube carcinoma (PFTC), magnetic resonance diffusion-weighted imaging, ADC values of fallopian tube tumours.

\section{Introduction}

Primary fallopian tube carcinoma (PFTC) is the rarest form of female genital malignancy. Its frequency is estimated to be less than $1-2 \%$. It is mostly diagnosed in the period from the fourth to sixth decade of life [1,2]. The germ-line BRCA1 and BRCA2 mutations are the only documented risk factors related to its aetiology. Some papers have described the theory that high-grade serous ovarian carcinoma and peritoneal carcinoma may originate from occult high-grade serous carcinoma in the fallopian tubes $[3,4]$. Diagnostics carried out for suspected adnexal masses includes transvaginal ultrasound, computed tomography (CT), and magnetic resonance imaging (MRI) of the pelvis and abdomen, but the vast majority of PFTC are recognised intraoperatively. The treatment of PFTC is based on debulking surgery and adjuvant platinum-based chemotherapy, similar to ovarian cancer $[5,6]$.

Correspondence address:

Paweł Derlatka, MD, PhD, Assoc. Prof., $2^{\text {nd }}$ Department of Obstetrics and Gynaecology, Medical University of Warsaw, 2 Karowa St., 00-315 Warsaw, Poland,

phone: +48 2259664 21, e-mail: pawel.derlatka@wum.edu.pl

Authors' contribution:

A: Study design, B: Data collection, C: Statistical analysis, D: Data interpretation, E: Manuscript preparation, F: Literature search, G: Funds collection 
MR imaging is the established modality for the diagnosis of adnexal malignancies. This technique can also be used to determine the source or extension of disease. Advances in MRI technology, including improvement of diffusion-weighted imaging technique (DW imaging), could increase the sensitivity of MRI for differentiating tumours of the fallopian tube and ovary, especially in the early stages. Previous studies have shown the efficacy of DW images to detect different pelvic malignancies, e.g. ovarian [7]. There is lack of standardisation of DW image acquisition (choice of different $b$ values) and calculation of the apparent diffusion coefficient (ADC).

The aim of this study was to assess the accuracy of DW imaging for the detection of PFTC in patients with suspected adnexal masses, and to evaluate ADC values of fallopian tube tumours.

\section{Material and methods}

The study group consisted of seven women aged from 48 to 69 years with postoperative histopathologic diagnosis of PFTC. In all cases serous high-grade carcinomas were confirmed. According the FIGO staging, four patients were at stage I, one at stage II, and two at stage III of PFTC.

To recognise characteristic findings for PFTC the retrospective analysis of preoperative MR imaging was performed.

\section{Magnetic resonance imaging}

The group of patients underwent MR imaging of the abdomen and pelvis at our MR Lab. All MR imaging was performed with a $1.5 \mathrm{~T}$ clinical whole-body MR system
(MAGNETOM Avanto; Siemens AG, Erlangen) using the Spine Matrix coil for full coverage of the abdomen and pelvis. Based on the results of the above imaging, suspected adnexal masses were recognised.

MRI protocol for the detection of the abdominal and pelvic lesions consisted of turbo spin-echo (TSE) T2-weighted images, fat-suppressed T2-weighted, T2TIRM, DW EPI, pre- and post-contrast dynamic 3D T1 GRE in transverse orientation. The most imported of the applied parameters of MR imaging are shown in Table 1.

Pelvic axial DW images were acquired using the same multi-slice EPI sequence for all cases: $30 \times 6 \mathrm{~mm}$ slices; $380 \times 380 \mathrm{~mm}$ FOV; $128 \times 96$ matrix; TR $=3800 \mathrm{~ms}$; TE $=73 \mathrm{~ms}$; with diffusion weightings of $0,50,100,150,200$, 400, 800, and $1200 \mathrm{~s} / \mathrm{mm}^{2}$.

Gadobutrol (Gadovist, Bayer Schering) was administered at a dose of $0.1 \mathrm{ml} / \mathrm{kg}$, immediately followed by a bolus of $20 \mathrm{ml}$ of $0.9 \% \mathrm{NaCl}$.

\section{Image analysis}

Regions of interest (ROI) were outlined in a MultiModality Workplace Station (Siemens Medical Solution) by a radiologist with experience in pelvic MR imaging, who documented the character of adnexal masses on DW images and ADC maps.

Subsequently, freehand ROI were drawn on the ADC and all $b$ value images by using the T2-weighted images for guidance. ROI included as large a part of the solid lesion as possible, avoiding areas of necrosis, partial volume effect, and artefacts. ROI was copied and pasted from DW image to proper ADC map. The measurements on the ADC map were recorded. ADC was measured twice for

Table 1. Parameters of applied magnetic resonance sequences

\begin{tabular}{|c|c|c|c|c|c|}
\hline Parameter & T2 TSE & T2 TSE; Fat-Sat & DW EPI & T2 TIRM & 3D T1 GRE \\
\hline Repetition time (ms) & 4250 & 2110 & 3800 & 6100 & 3.05 \\
\hline Echo time (ms)] & 117 & 123 & 73 & 39 & 1.13 \\
\hline Flip angle (deg.) & 137 & 150 & 90 & 150 & 10 \\
\hline Turbo factor & 51 & 51 & - & 9 & - \\
\hline EPI factor & - & - & 96 & - & - \\
\hline ¡PAT factor & - & 2 & 2 & - & 2 \\
\hline Plane & Axial & Axial & Axial & Axial & Axial \\
\hline Number of signal averages & 1 & 1 & 4 & 1 & 1 \\
\hline FOV (mm) & 360 & 360 & 360 & 360 & 360 \\
\hline Rectangular FOV (\%) & 75 & 100 & 75 & 75 & 75 \\
\hline Matrix & $384 \times 512$ & $256 \times 256$ & $96 \times 128$ & $288 \times 384$ & $156 \times 288$ \\
\hline Slice thickness (mm) & 5 & 5 & 6 & 5 & 3 \\
\hline Respiratory triggering & No & Yes & No & No & No \\
\hline Breath-hold & No & Yes & No & No & No \\
\hline
\end{tabular}

EPI - echo-planar imaging, FOV - field of view 


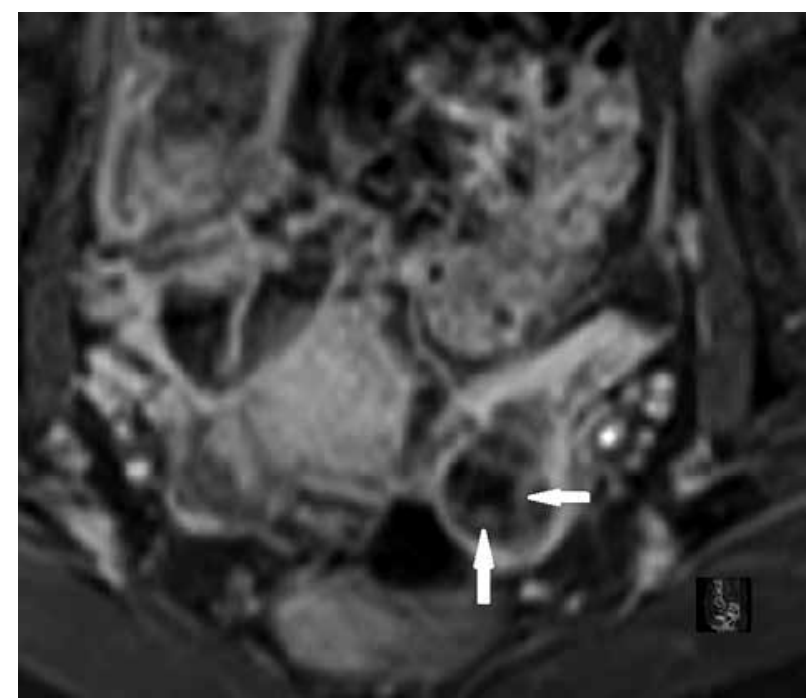

Figure 1. Primary fallopian tube carcinoma. Axial T1-weight fat-sat magnetic resonance imaging. The moderate and heterogenous enhancement of solid parts of the tumour (arrows)

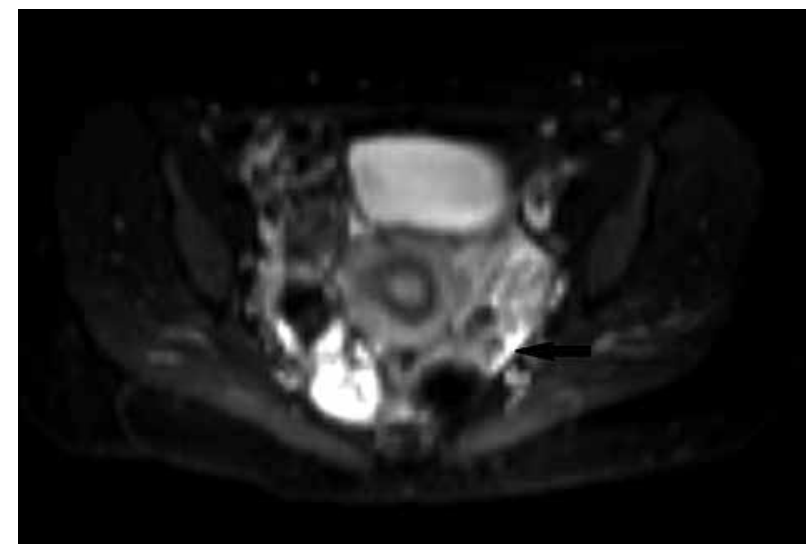

Figure 3. Primary fallopian tube carcinoma. Patient 2. Diffusion-weighted imaging $b 100 \mathrm{~s} / \mathrm{mm}^{2}$. The part of the solid tumour of fallopian tube with high signal intensity (arrow)

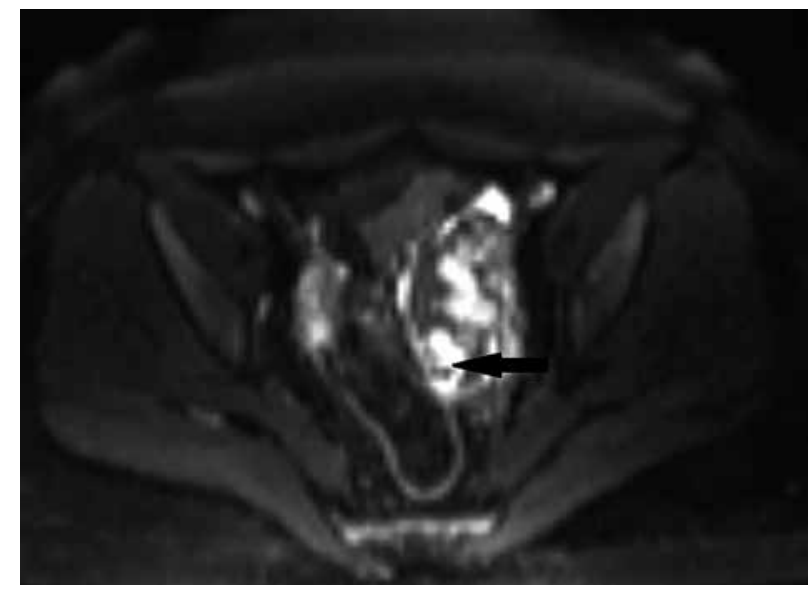

Figure 5. Primary fallopian tube carcinoma. Patient 2. Diffusion-weighted imaging $b 800 \mathrm{~s} / \mathrm{mm}^{2}$. The part of the solid tumour of fallopian tube with high signal intensity (arrow)

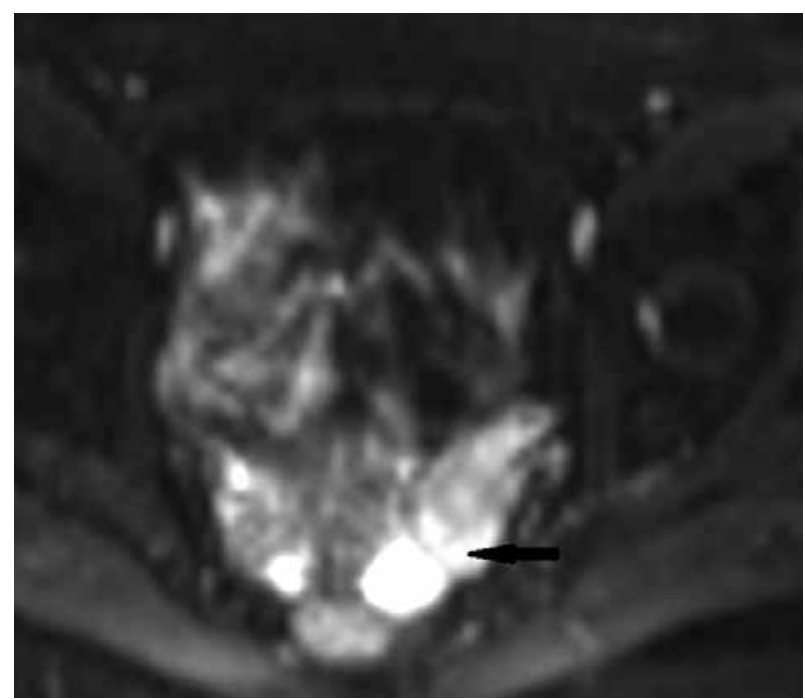

Figure 2. Primary fallopian tube carcinoma. Patient 1. Diffusion-weighted imaging $b 100 \mathrm{~s} / \mathrm{mm}^{2}$. The part of the solid tumour of fallopian tube with high signal intensity (arrow)

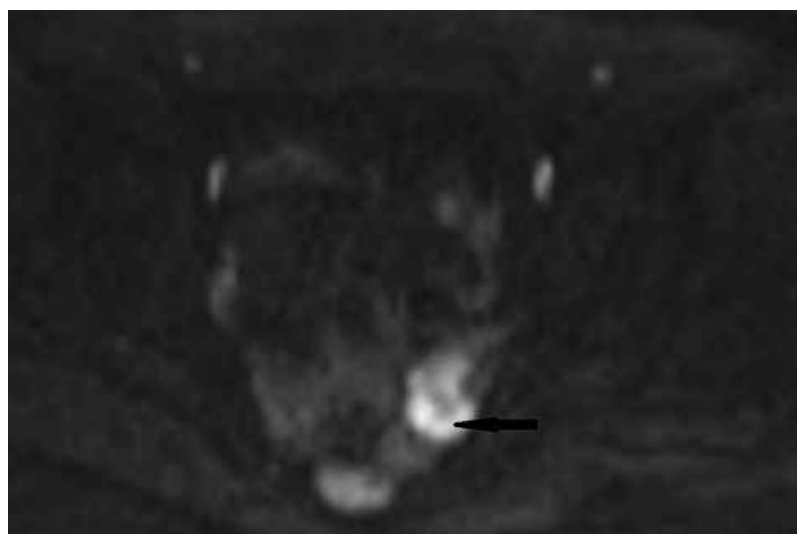

Figure 4. Primary fallopian tube carcinoma. Patient 1. Diffusion-weighted imaging $b 800 \mathrm{~s} / \mathrm{mm}^{2}$. The part of the solid tumour of fallopian tube with high signal intensity (arrow)

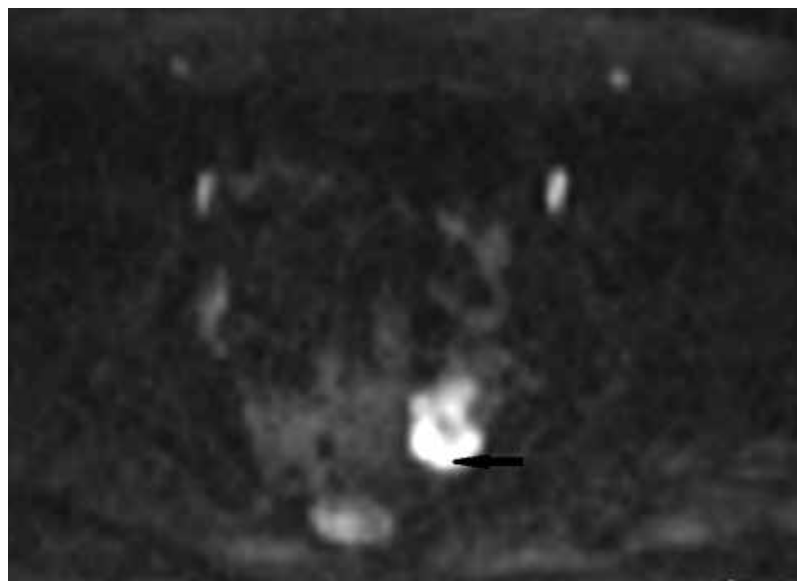

Figure 6. Primary fallopian tube carcinoma. Patient 1. Diffusion-weighted imaging $b 1200 \mathrm{~s} / \mathrm{mm}^{2}$. The part of the solid tumour of fallopian tube with high signal intensity (arrow) 


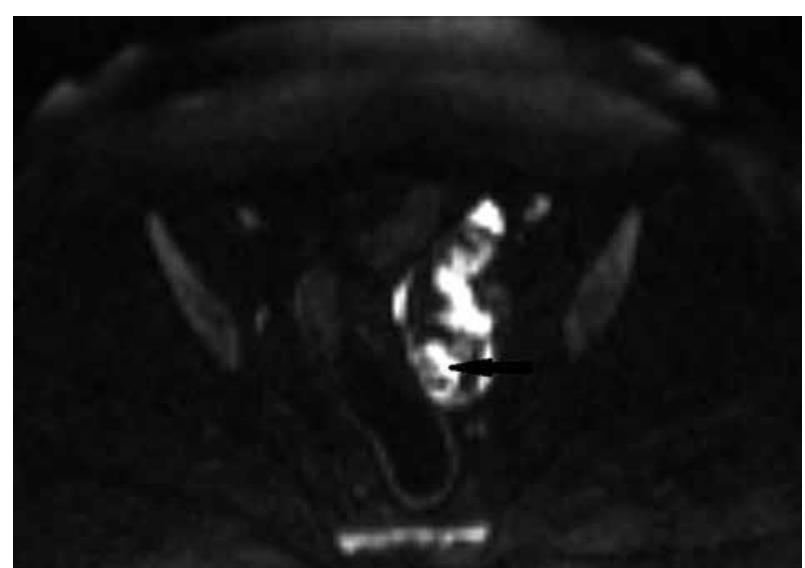

Figure 7. Primary fallopian tube carcinoma. Patient 2. Diffusion-weighted imaging $b 1200 \mathrm{~s} / \mathrm{mm}^{2}$. The part of the solid tumour of fallopian tube with high signal intensity (arrow)

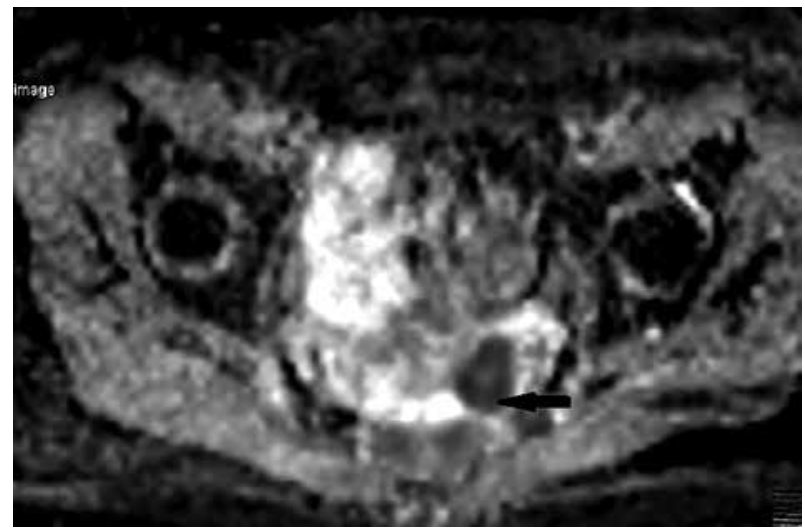

Figure 8. Primary fallopian tube carcinoma. Patient 1. Diffusion-weighted imaging. $A D C$ map, restricted diffusion inside the part of solid tumour (arrow)

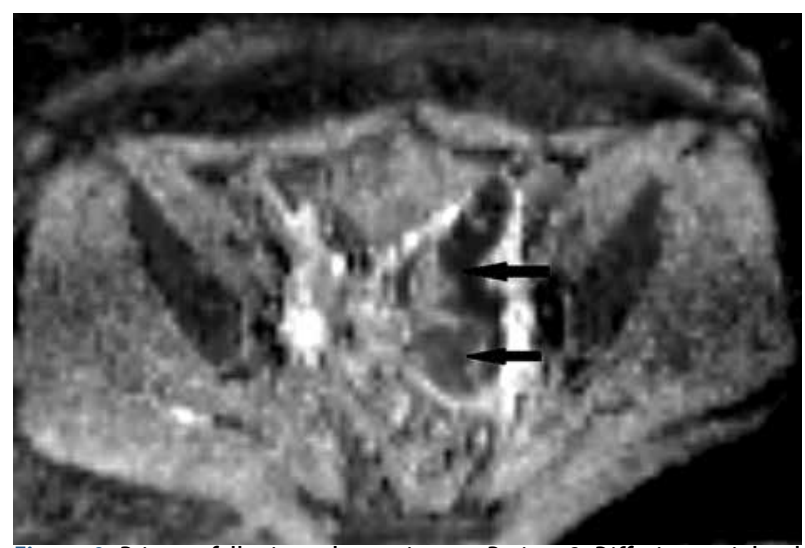

Figure 9. Primary fallopian tube carcinoma. Patient 2. Diffusion-weighted imaging. $A D C$ map, restricted diffusion inside the part of solid tumour (arrow)

each solid lesion and the results were averaged. Knowing postoperative histopathological diagnosis of PFTC, the following control ADC measurement sites were chosen as normal ovary on the tumour side in three separate points. If the ovary on the tumour side was impossible to identify, an ADC measurement was performed for the ovary on the opposite side to the tumour.
ADC values were calculated by mono-exponential regression with the following formula: $\mathrm{S}=\mathrm{S}_{0} \times \exp (-b \times \mathrm{DC})$, where $S$ is the signal intensity after application of the diffusion gradient, and $S_{0}$ is the signal intensity at $b=0 \mathrm{~s} / \mathrm{mm}^{2}$. Eight $b$ values were applied for ADC calculation.

\section{Statistical analysis}

All statistical analyses were performed using the STATISTICA v.12 (Statsoft, Tulsa, USA) software package. Comparisons of mean ADC and mean $b$-value images ( $b 50$, $100,150,200,400,800,1200 \mathrm{~s} / \mathrm{mm}^{2}$ ) between solid parts of fallopian tube tumour and normal ovarian tissue were analysed using an unpaired two-tailed $t$-test; $p<0.05$ indicated a statistically significant difference.

\section{Results}

In all seven patients with PTFC unilateral tumour was found. The largest tumour diameter ranged from $18 \mathrm{~mm}$ to $130 \mathrm{~mm}$. MRI shown a sausage-like shape tumour in five patients (71\%) and irregular polycystic lesion in two cases $(29 \%)$. The signal the solid parts of the fallopian tube tumour was homogenous and hyperintense in T2-weighted images and demonstrated enhancement on T1-weighted images in all patients (Figure 1).

On all DW images (with $b$ values of $0,50,100,150$, $200,400,800$, and $1200 \mathrm{~s} / \mathrm{mm}^{2}$ ) the mean signal intensities of solid parts of tumour were significantly higher than the mean signal intensities of normal ovarian tissue $(p=0.0001)$ (Figures 2-7). Mean ADC values of tumour tissue were significantly lower than those of reference ovary $(p=0.0001)$. There were no statistically significant differences between the eight $b$ values applied for ADC calculations (Figures 8 and 9).

In three patients in FIGO stage II and III extratumoural lesions were found on DW-images (peritoneal and omental implants).

\section{Discussion}

PFTC represent less than $1 \%$ of all female genital malignancies. Pathology remains the mainstay for diagnosis of PFTC. Serous carcinoma is the most common histological type of tubal cancer. These tumours are identical to their ovarian counterparts [1].

Clinically it resembles ovarian cancer, and ovarian tumour is the usual preoperative recognition. The tubal carcinoma is rarely recognised preoperatively and is usually first appreciated during the operation or by a pathologist $[5,6]$.

Due to its very rare occurrence, previous original reports of diagnosis of PFTC relate to groups from a few to a dozen patients. Imaging routinely carried out for suspected adnexal masses includes transvaginal ultrasound, CT scan, and MR imaging of the abdomen and pelvis. The appearance of PFTC can be characteristic: an adnexal 
tubular cystic structure, representing hydrosalpinx, and containing papillary projections or solid mural nodule [8].

MR imaging is particularly helpful in the initial evaluation of adnexal malignancies, due to its multiplanar capability with superior soft tissue contrast. Until now the differential diagnosis in MRI was based on morphological feature as a typical small sausage-like solid unilateral mass. On MRI hydrosalpinx appears as simple serous fluid, homogeneously hyperintense on T2-weighted images and hypointense on T1-weighted images [8]. We obtained these results in our group. If protein or haemorrhagic fluid is present, it appears heterogeneously hyperintense on T2-weighted images and hyperintense on T1-weighted images. The solid tumour component of PFTC is homogeneously or heterogeneously iso- or hyperintense on T2-weighted images, hypointense on T1-weighted images, and demonstrates enhancement $[9,10]$. In some papers the authors suggest that the enhancement of solid parts is mild to moderate, heterogenous, and uncharacteristic [11,12]. However, the diagnosis is still based on morphological features.

We tried to find specific features of PFTC in DW imaging. On our exams (with $b$ values of $0,50,100,150$, $200,400,800$, and $1200 \mathrm{~s} / \mathrm{mm}^{2}$ ) the diffusion restriction of solid parts of the tumour were significantly higher than the mean signal intensities of normal ovarian tissue $(p=0.0001)$. There is no border b value specific to PFTC. It is the different PFCT from the ovarian carcinoma, where the most ovarian malignancies show diffusion restriction at high $b$ levels $(>300)[13,14]$.

The small number of patients is an important limitation of this study, but we must take into account that PFTC is a very rare tumour. However, our results should be regarded as preliminary in terms of sensitivity and specificity of DW imaging for the differential diagnosis of PFTC and should be confirmed in a multicentre study.

\section{Conclusions}

Preoperative diagnostics of PFTC is difficult and is based mainly on morphological features. Previous research did not show characteristics of PFTC in post-contrast dynamic imaging. In our material a clear increase in signal intensity in DW imaging occurs independently of the $b$ value.

\section{Conflict of interest}

The authors report no conflict of interest.

\section{References}

1. Alvarado-Cabrero I, Stolnicu S, Kiyokawa T, et al. Carcinoma of the fallopian tube: results of a multi-institutional retrospective analysis of 127 patients with evaluation of staging and prognostic factors. Ann Diagn Pathol 2013; 17: 159-164.

2. Nik NN, Vang R, Shih I-M, et al. Origin and pathogenesis of pelvic (ovarian, tubal, and primary peritoneal) serous carcinoma. Annu Rev Pathol Mech Dis 2014; 9: 27-45.

3. Dietl J. Revisiting the pathogenesis of ovarian cancer: the central role of the fallopian tube. Arch Gynecol Obstet 2013; 289: 241-246.

4. Seidman JD, Zhao P, Yemelyanova A. "Primary peritoneal" highgrade serous carcinoma is very likely metastatic from serous tubal intraepithelial carcinoma: assessing the new paradigm of ovarian and pelvic serous carcinogenesis and its implications for screening for ovarian cancer. Gynecol Oncol 2011; 120: 470-473.

5. Gadducci A, Landoni F, Sartori E, et al. Analysis of treatment failures and survival of patients with fallopian tube carcinoma: a cooperation task force (CTF) study. Gynecol Oncol 2001; 81: 150-159.

6. Pectasides D, Pectasides E, Economopoulos T. Fallopian tube carcinoma: a review. Oncologist 2006; 11: 906-912.

7. Kyriazi S, Collins DJ, Morgan VA, et al. Diffusion-weighted imaging of peritoneal disease for noninvasive staging of advanced ovarian cancer. Radiographics 2010; 30: 1269-1285.
8. Shaaban AM, Rezvani M. Imaging of primary fallopian tube carcinoma. Abdom Imaging 2013; 38: 608-618.

9. Rezvani M, Shaaban AM. Fallopian tube disease in the nonpregnant patient. Radiographics 2011; 31: 527-548.

10. Hosokawa C, Tsubakimoto M, Inoue $\mathrm{Y}$, et al. Bilateral primary fallopian tube carcinoma: findings on sequential MRI. Am J Roentgenol 2006; 186: 1046-1050.

11. Gomes FV, Dias JL, Lucas R, et al. Primary fallopian tube carcinoma: review of MR imaging findings. Insights Imaging 2015; 6: 431-439.

12. Ma FH, Cai SQ, Qiang JW, et al. MRI for differentiating primary fallopian tube carcinoma from epithelial ovarian cancer. J Magn Reson Imaging 2015; 42: 42-47.

13. Sala E, Kataoka MY, Priest AN, et al. Advanced ovarian cancer: multiparametric MR imaging demonstrates response and metastasis-specific effects. Radiology 2012; 263: 149-159.

14. Lindgren A, Anttila M, Rautiainen S, et al. Primary and metastatic ovarian cancer: characterization by 3.01 diffusion-weighted MRI. Eur Radiol 2017; 27: 4002-4012. 